\title{
Tracheobronchial amyloidosis: A surgical disease with long-term consequences
}

\author{
Kevin A. Dahl, BA, ${ }^{a}$ Kemp H. Kernstine, MD, PhD, ${ }^{a}$ Timothy L. Vannatta, MD, ${ }^{\text {a }}$ Mark W. Karwal, MD, ${ }^{\text {b }}$ \\ Karl W. Thomas, MD, ${ }^{b}$ and Daniel F. Schraith, MD, ${ }^{c}$ lowa City, lowa
}

A myloidosis results from abnormal protein deposition and accumulation in extracellular spaces. ${ }^{1}$ The multiple distinct forms of this disease have heterogeneous clinical manifestations and variable patterns of organ involvement. Amyloidosis might be hereditary or acquired, localized, or systemic and can range from an incidental asymptomatic finding to a lethal disorder. The diseases are distinguished on a molecular level by the type of peptide subunits that compose abnormally accumulated protein fibrils. Although these peptide fibrils differ, all amyloid fibrils adopt a similar ultrastructure rich in $\beta$-sheet content. The fibril deposits also have a common association with certain glycosaminoglycans (GAGs), notably heparin and dermatan sulphate. Normal plasma proteins, such as serum amyloid P (SAP), function to stabilize the amyloid fibril. ${ }^{1} \mathrm{We}$ present a case of severe tracheobronchial amyloidosis (TBA) with the results of our evaluation and management strategies.

\section{Clinical Summary}

A 49-year-old white man had a 6-year history of progressive dyspnea and productive cough. He had an accelerated symptom progression over the prior 6 months and also noted the new onset of intermittent malaise, myalgias, arthralgias, fever, chills, and night sweats. He had been treated with parenteral and oral antibiotics, as well as a variety of inhaled bronchodilators for suspected pulmonary infections. Because he experienced only transient improvement, a combination of prednisone, fluticasone, albuterol, salmeterol, and ipratropium were added, but these did not improve his symptoms.

Past medical history was remarkable for recurrent pneumonia and bronchitis treated empirically. He had a 30 pack-year smoking history but quit smoking 11 years previously.

Hepatic, renal, and thyroid function test results were all normal, as were electrolyte levels and hemography results. Pulmonary function tests were as follows: forced vital capacity, 4.95 L (101\% of predicted value); forced expiratory volume in 1 second, $4.02 \mathrm{~L}$

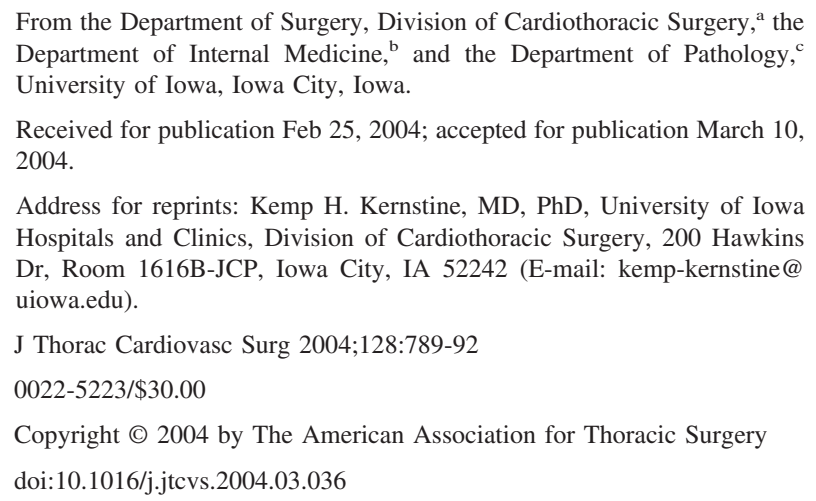

(88\% of predicted value); total lung capacity, $7.06 \mathrm{~L}$ (97\% of predicted value); residual volume, $2.25 \mathrm{~L}$ ( $82 \%$ of predicted value); and lung diffusion of carbon monoxide, 30.7 (99\% of predicted value). There was no improvement with a bronchodilator. The results of chest radiographs 1 to 2 years previously were normal. Chest radiographs on presentation suggested significant narrowing of the left main bronchus, with no infiltrates or evidence of congestion. Chest computed tomography (CT; Figure 1) showed soft tissue calcification of the proximal left lingular and upper lobe segment bronchus, suggesting a mass, potentially a broncholith.

Bronchoscopy (Figure 2) revealed an edematous left upper lobe airway with friable mucosa and lesser involvement of the left carina and left lower lobe bronchial mucosa. The entire left tracheobronchial tree appeared to have narrowing and stenosis, with the left upper lobe most severe. Bronchoalveolar lavage of the left upper lobe did not identify any tumor cells. Histologic examination of the mucosal biopsy specimen revealed fragments of bone and cartilage, with calcification, degenerated respiratory epithelium, amorphous debris, and mild acute and chronic inflammation. These findings were nonspecific but raised the possibility of pulmonary hematoma, broncholith, or possibly teratoma. No evidence of carcinoma or granulomatous disease was found in the superficial endobronchial biopsy specimens. However, these 2 clinical entities remained in the differential diagnosis.

Considering the patient's age, smoking history, chest CT findings, and progressive symptoms, a paraneoplastic syndrome could not be ruled out. The results of bone scanning and head magnetic resonance imaging were normal. A whole-body positron emission tomogram was also normal, except for a single focus of left hilar activity with a standardized uptake value of 3.5. Surgical consultation was obtained, and the possibility of left pneumonectomy was discussed with the patient and our health care team given the atypical clinical presentation and the presence of systemic symptoms.

Cervical and left anterior mediastinoscopy revealed no metastatic disease. Exploratory left thoracotomy showed no pleural process, and the hilum appeared normal. On palpation, however, there was a deep hilar mass surrounding the left main stem bronchus. Hilar dissection exposed associated adenopathy. Biopsy of the mass and nodes showed inflammation on frozen section. The fissure was opened, and encountered nodes underwent biopsy, demonstrating no malignancy. The upper lobe airway was firm and bone-like. This bronchus was longitudinally incised along its membranous portion. Intraluminally, very firm inflammation almost completely obliterated the entire upper lobe bronchus. The visualized scarring and bronchial occlusion was so severe and solid that it was unlikely the patient would benefit from less invasive airway techniques, such as laser tissue ablation, balloon bronchoplasty, or stenting. Given the pathology, the patient would remain at risk for recurrent pneumonias without definitive treat- 


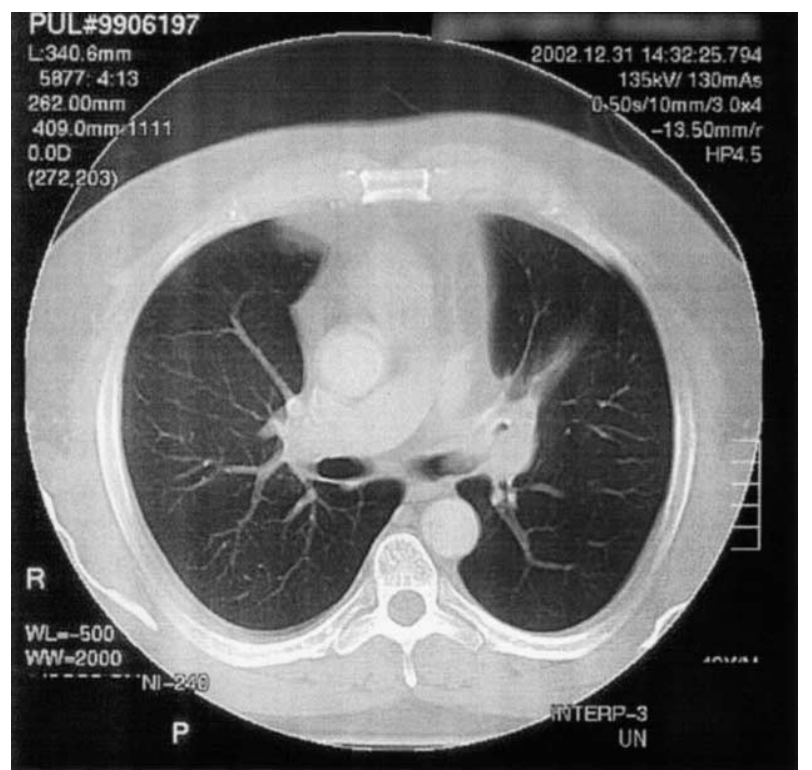

Figure 1. Contrast-enhanced chest CT scan just inferior to the tracheal bifurcation. A soft tissue mass surrounds the left main stem bronchus, extending into the bronchial lumen.

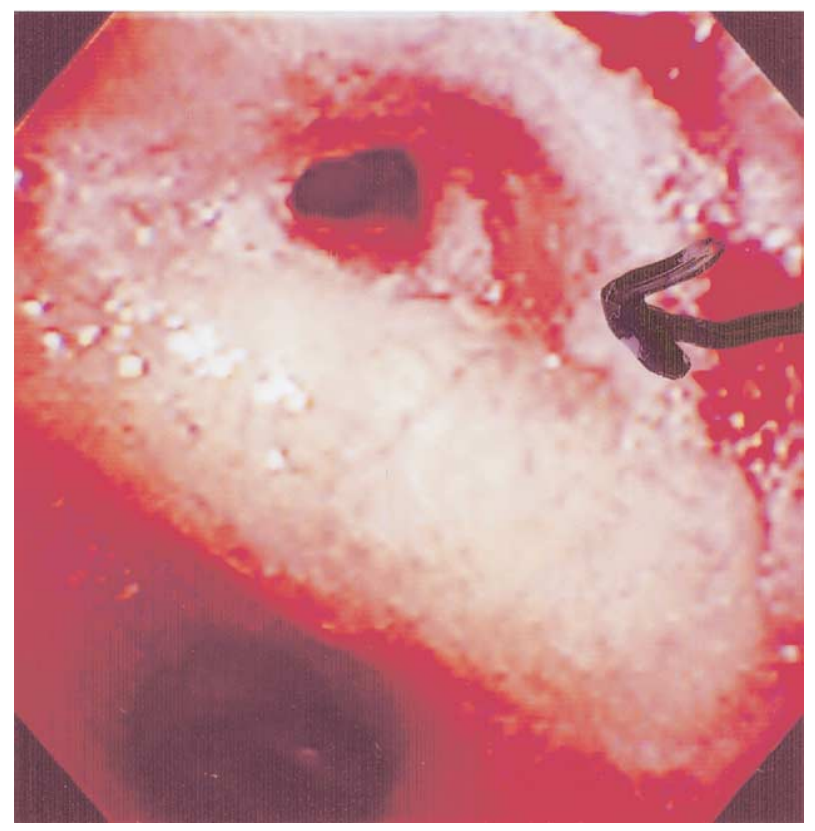

Figure 2. On bronchoscopy, this is the appearance of the left upper lobe bronchus. The bronchial subdivisions are edematous and narrowed.

ment. After significant deliberation, a left upper lobectomy was performed, leaving the origin of the left upper lobe bronchus open to allow examination of the left main stem bronchial lumen. The severely obstructive and circumferential process extended into both the left lower lobe and the left main bronchi. It appeared to

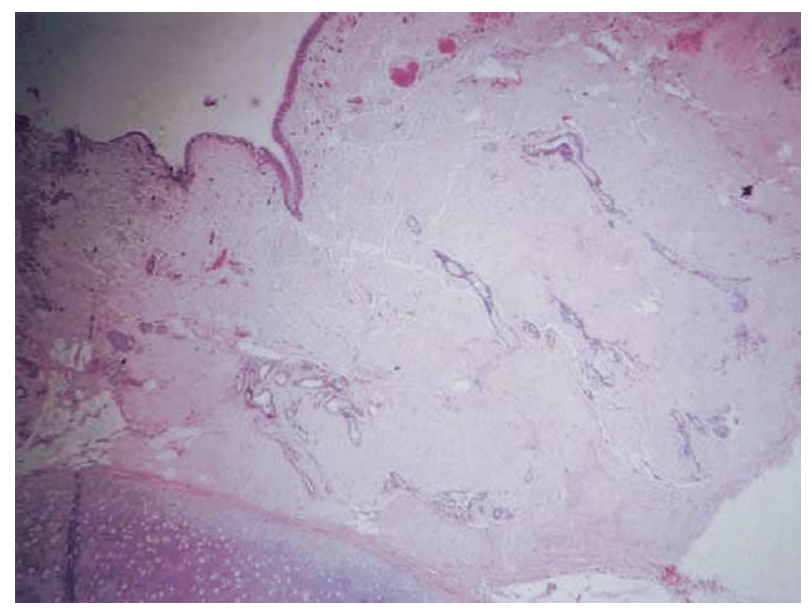

Figure 3. Histologic sample of the bronchus. Normal ciliated respiratory mucosal lining at the upper left, bronchial cartilage at the lower left, and submucosa widely distended with amorphous pink material, amyloid, is shown.

stop at the carina. Histology of the left upper lobe bronchial margin demonstrated calcification but no tumor. The inflammatory process and obstruction in the left main stem and lower lobe were so severe that it was believed also to be irreversible. Thus high transection of the left main stem bronchus was performed, completing a left pneumonectomy. The postoperative course was uneventful.

The final pathology revealed marked and diffuse expansion of the bronchial submucosa by amorphous pink material, which was consistent with amyloid (Figures 3 and 4). Congo red staining confirmed the presence of amyloid (Figure 5). Areas of osseous metaplasia were also noted.

Subsequent clinical evaluation included a fat pad aspirate and bone marrow biopsy, and immunofixation studies of serum and urine demonstrated no evidence of systemic amyloidosis. Six months after pneumonectomy, the patient continued to have systemic nonpulmonary symptoms, including malaise, myalgias, arthralgias, and failure to thrive.

\section{Discussion}

Amyloid is not a single, molecularly distinct protein; it is more accurately defined as a group of molecularly diverse proteins composed of nonbranching linear fibrils arranged in sheets. The heterogeneity of amyloidosis is attributable in part to the diversity of fibril precursor peptide units that constitute amyloid. Common to all amyloid protein is an ultrastructure rich in $\beta$-sheet content and structural associations with GAGs. Plasma proteins, notably SAP, stabilize the long linear amyloid fibrils. ${ }^{1}$ The majority of amyloidosis, both systemic and localized types, consist of the light chain-associated subunit. Other amyloid types also exist and are composed of a spectrum of precursor peptide units ${ }^{1}$; a detailed discussion of the complex biochemistry of amyloid proteins is beyond the scope of this discussion.

Amyloid deposition can occur in the lung in the setting of primary or secondary systemic disease or can be limited to the lung. In primary systemic amyloidosis, amyloid deposits in the lung in $30 \%$ to $90 \%$ of cases, usually in a diffuse distribution. 


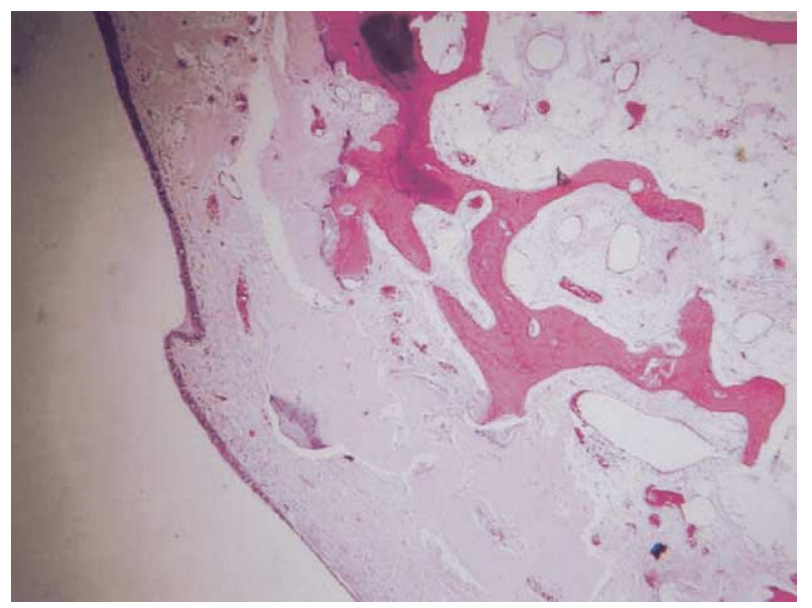

Figure 4. Histologic sample of bronchus demonstrating submucosa. Seromucinous glands and blood vessels are entrapped in the amyloid. With normal ciliated respiratory mucosal lining at left, osseous metaplasia is present in the center as pink branching trabeculae. The submucosa is widely distended with amorphous pink amyloid.

Secondary amyloidosis can occur in a host of chronic inflammatory diseases, including Crohn disease, ankylosing spondylosis, tuberculosis, and bronchiectasis. When amyloid deposition is limited to the respiratory tract, it is usually in one of 3 patterns of distribution: (1) nodular parenchymal; (2) tracheobronchial; and (3) diffuse alveolar septal, also called diffuse parenchymal.

TBA is characterized by amyloid deposits primarily in the trachea and large bronchi, with extension at times into segmental bronchi. Submucosal vessels are frequently involved. ${ }^{2}$ TBA typically presents in patients in their 40s and 50s, with reports ranging from age 27 to 85 years. ${ }^{1,3}$ Common presenting symptoms include chronic cough, dyspnea, wheezing, hemoptysis, and recurrent pneumonia. ${ }^{1,3}$ The pulmonary symptomatology help to distinguish it from other forms of pulmonary amyloidosis because patients with primary systemic amyloidosis rarely present with pulmonary symptoms, and the nodular parenchymal form is often an incidental finding. ${ }^{2}$ Radiographic findings of TBA include focal or diffuse airway wall thickening, a localized nodule within the airway lumen, or possible airway obstruction with atelectasis, postobstructive pneumonia, and distal lung air trapping. ${ }^{2}$ Grossly, the airway appears thickened and irregular, with waxy, firm, or gritty deposits and yellow, gray, tan, or white coloring. ${ }^{2}$ TBA has been confused with tracheobronchopathia osteoplastica, which is characterized by calcified or cartilaginous submucosal nodules but without amyloid deposition. ${ }^{1}$ The clinical presentation can be similar to that of tracheobronchopathia osteoplastica, lymphoproliferative disorders, primary or metastatic neoplasms, relapsing polychondritis (which involves the laryngeal and tracheal cartilages in $>50 \%$ of patients), sarcoidosis, and Wegener granulomatosis. ${ }^{2,3}$ Lymphoproliferative disorders and neuroendocrine tumors, especially carcinoids and small cell carcinomas, might develop tracheobronchial amyloid deposits, although the airway amyloid is minimal compared with that seen in primary TBA. $^{2}$

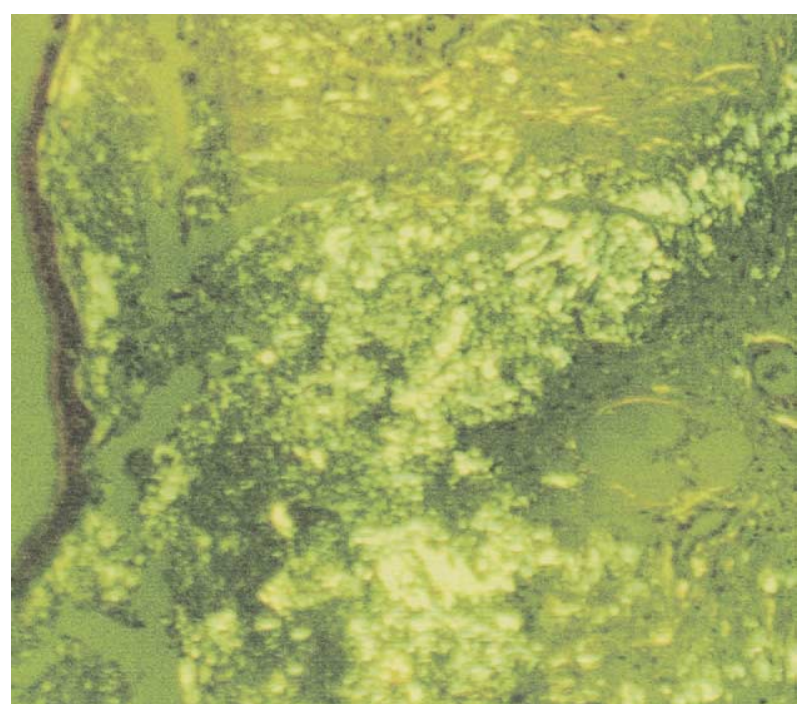

Figure 5. Congo Red stain of the bronchus histology. Amyloid demonstrates the characteristic apple green birefringent optical quality with polarized light.

Diagnosis of TBA requires histologic confirmation; Congo Red stain highlights the amyloid with a characteristic apple green birefringence with polarized light. ${ }^{1}$ Some have advocated immunohistochemical analysis of the amyloid deposits to determine the fibril type. ${ }^{1}$ Although the light chain-associated type is typical in TBA (as well as primary systemic amyloidosis), other rarer fibril types can be detected and might have therapeutic implications. $^{4}$

A comprehensive evaluation, including radiolabeled SAP scan, CT scan of the thorax, echocardiography, and bone marrow, serum, and urine studies need to be performed to accurately differentiate localized from systemic disease. ${ }^{4}$ Radiolabeled SAP localizes amyloid deposits in proportion to the quantity of amyloid present, thus assisting diagnosis, quantification, and monitoring of amyloid deposits. Electrocardiography and echocardiography are fairly sensitive and specific for cardiac involvement, which commonly occurs in systemic amyloidosis and is a major prognostic determinant. ${ }^{1}$ Protein electrophoresis studies on serum and urine can help detect an underlying lymphoproliferative disorder with an associated monoclonal gammopathy. However, up to $10 \%$ of patients with limited pulmonary amyloidosis might demonstrate circulating monoclonal proteins. $^{2}$

Although a localized disease process, overall survival in TBA is only $31 \%$ to $43 \%$ at 4 to 6 years. ${ }^{2}$ In contrast, pulmonary amyloidosis is generally a benign process, with resection being both diagnostic and curative. Therefore differentiating these 2 entities has profound prognostic implications. ${ }^{2}$

There is no proved pharmacologic therapy for TBA. Treatment options include bronchoscopic or surgical resection, as well as laser therapy. ${ }^{1}$ Management of TBA is largely dictated by the degree of symptoms. Treatment might involve intermittent bronchoscopic or surgical resection, carbon dioxide laser ablation, and Nd:YAG laser therapy. ${ }^{1}$ Repeated bronchoscopic treatment is thought to be safer than open surgical intervention. ${ }^{1}$ However, 
extensive airway involvement requires open resection, as reported herein. There are anecdotal reports of successful treatment of pulmonary amyloidosis with dimethylsulfoxide. ${ }^{2}$ One study reports 2 cases of endobronchial amyloidosis treated with an $\mathrm{Nd}$ : YAG laser, resulting in patency of the involved lung segments 10 and 16 months later. ${ }^{5}$ External beam radiation therapy has also been used, with clinical and functional improvement noted after 18 months of follow-up. ${ }^{6}$

Research is currently underway seeking to prevent amyloid deposition by stabilizing the amyloid precursor proteins in their normal conformation, inhibiting fibril propagation, enhancing fibril degradation, and developing GAG mimetics and SAP binding inhibitors. Competitive inhibition of SAP binding targets the stabilizing role of SAP in the amyloid fibril. Pepys and colleagues ${ }^{7}$ recently reported SAP binding by Ro 63-8695 could both reduce plasma SAP levels and competitively inhibit SAP-amyloid binding. The mechanism of action involves cross-linking pairs of pentameric SAP molecules, thus creating a decameric configuration rapidly cleared by the liver.

TBA is a rare disease resulting from abnormal protein deposition within the airway submucosa. Despite surgical resection and treatment of the obstructive pneumonia, long-term survival is poor. Exclusion of systemic amyloidosis is necessary in the evaluation of a patient with pulmonary amyloid deposition. There is no cure, although laser therapy, external beam radiation, and pharmacologic SAP binders might potentially improve the outlook for these patients.

\section{References}

1. Gillmore JD, Hawkins PN. Amyloidosis and the respiratory tract. Thorax. 1999;54:444-51.

2. Travis WD, Colby TV, Koss MN, et al. Non-neoplastic disorders of the lower respiratory tract. Atlas of nontumor pathology: 2. Washington, DC: American Registry of Pathology; 2002, p. 873-81.

3. Farrell M, McAdams HP, Erasmus JJ. A 27-year-old woman with cough, dyspnea, and wheezing. Chest. 1999;116:557-9.

4. Shah PL, Gillmore JD, Copley SJ, et al. The importance of complete screening for amyloid fibril type and systemic disease in patients with amyloidosis in the respiratory tract [abstract]. Sarcoidosis Vasc Diffuse Lung Dis. 2002;19:134-42.

5. Madden BP, Lee M, Paruchuru P. Successful treatment of endobronchial amyloidosis using Nd: YAG laser therapy as an alternative to lobectomy. Monaldi Arch Chest Dis. 2001;56:27-9.

6. Monroe AT, Walia R, Zlotecki RA, Jantz MA. Tracheobronchial amyloidosis, a case report of successful treatment with external beam radiation therapy. Chest. 2004;125:784-9.

7. Pepys MB, Herbert J, Hutchinson WL, et al. Targeted pharmacological depletion of serum amyloid $\mathrm{P}$ component for treatment of human amyloidosis. Nature. 2002;417:254-9. 